\title{
An extended pair tunneling model: Studies on bilayer splitting and some superconducting state properties
}

\author{
A. N. Das ${ }^{\star}$ and Biplab Chattopadhyay ${ }^{\dagger}$ \\ Saha Institute of Nuclear Physics, \\ 1/AF Bidhannagar, Calcutta 700 064, India \\ PACS Numbers: 74.20.-z, 74.62.-c, 74.62.Dh
}

Keywords: High temperature cuprate superconductors, Interlayer tunneling, Pseudogap, Photoemission intensity.

^e-mail: atin@cmp.saha.ernet.in; ${ }^{\dagger}$ e-mail: biplab@cmp.saha.ernet.in 


\begin{abstract}
We consider an extended version of the pair tunneling model including interlayer single particle hopping (ISPH) as a complementary process to pair tunneling. The normal state gap, as found in cuprates, is taken to suppress the effective ISPH in conformity with the experimental observations, and this in turn enhances the pair tunneling process. The effective ISPH involves a probability factor $P$ for which we consider two choices and provide phenomenological arguments in favour of them. We address the issue of bilayer splitting by calculating the spectral density function and corresponding photoemission intensity curves and show that our calculations conform with the absence of bilayer splitting observed in ARPES experiments on Bi2212. We have also studied the temperature variation of the superconducting gap and ratio of the superconducting gap to $T_{c}$. Our results, obtained for both the choices of $P$, are reasonably in good agreement with those from experiments on cuprate superconductors. A linear $T$-dependent choice of $P$, however, yields a precise match to the experimantal data of the temperature varying superconducting gap.
\end{abstract}




\section{Introduction}

In order to explain various unusual properties of high- $T_{c}$ superconductors (HTS) a number of phenomenological models have been proposed. One such model is the interlayer pair tunneling model (ILPT), originally proposed by P. W. Anderson [1]. The model is based on the postulate that the coherent single particle tunneling between two $\mathrm{CuO}_{2}$ layers of HTS is blocked owing to strong electronic correlation effect, whereas tunneling of Cooper pairs between the layers is allowed. This delocalization process of the pairs gives rise to a substantial enhancement of pairing in each layer and raises the transition temperature [2]. An important outcome of the ILPT model is that it yields considerably higher $T_{c}$ for a bilayer system compared to that for a single layer system, even with a weak or moderate in-plane attractive interaction. Thus, the model can naturally explain higher values of $T_{c}$ in bi-layered or triple-layered systems compared to those of monolayer (in reality well separated layers) systems and obviates the need for a strong in-plane attractive interaction to achieve high $T_{c}$.

In the ILPT model of Anderson [1, 2] the coherent single particle tunneling between layers is assumed to be completely blocked, even though the bare (interlayer) hopping rate, as obtained from the electronic structure calculation is substantial [3]. The idea of absence of interlayer single particle tunneling originates from the anomalous c-axis transport properties of cuprates. For underdoped systems rapid increase in the c-axis resistivity with decreasing temperature and absence of a Drude peak in the optical conductivity along the c-axis [4] indicate absence of coherent single particle hopping between the layers [2]. Anderson argued that these anomalies are due to the strong electronic correlations present in cuprates and are characteristics of a non-fermi liquid behaviour. However, for sufficiently overdoped cuprates the $c$-axis resistivity shows metallic like temperature dependence as that in the $a b$-plane and the Drude peak appars in the $(c$-axis) optical conductivity spectrum [5, 6]. These facts suggest presence of interlayer single particle hopping (ISPH) in case of overdoped systems. In fact, it is generally beleived that the confinement of the electrons in $\mathrm{CuO}_{2}$ planes is a characteristic

feature of underdoped cuprates where correlation has its strongest effect. For overdoped 
systems, on the other hand, the effect of correlation becomes weaker, hence the charge carriers are expected to behave more like a Fermi liquid.

Recently, it has been established that a normal state pseudo gap $\left(E_{g}\right)$ exists in high- $T_{c}$ systems [7, 8]. This gap is momentum dependent and is given by

$$
E_{g}(k)=\frac{1}{2} E_{g}\left|\operatorname{Cos}_{x} a-\operatorname{Cos}_{y} a\right|
$$

where $a$ is the in-plane lattice constant and the half factor is introduced so that the maximum value of the gap for any particular doping becomes $E_{g}$. For underdoped systems $E_{g}$ is large, it decreases gradually with doping and becomes small or negligible for overdoped systems [7, 8, 9]. An interesting correlation has been observed between the normal state gap and the $c$-axis transport in cuprates. For underdoped system, where $E_{g}$ is large, the interlayer single particle hopping is negligible, while, for overdoped system, where $E_{g}$ is small, it is allowed. Hence, a relationship, that the normal state gap is connected with the suppression of the interlayer single particle hopping, could be inferred.

Motivated by the above observations and inference, recently we proposed an extended pair tunneling model [10, 11] involving both ISPH and interlayer pair tunneling [10, 11], which is an extension of Anderson's ILPT model [2]. We assume that the particles can tunnel between two layers via two channels: (i) single particle hopping and (ii) pair tunneling, and these two processes are complementary. We introduced a probability factor $(P)$ for the ISPH which decreases very fast with the increase of $E_{g}$ [10]. As a result, for underdoped systems where $E_{g}$ is large, the ISPH is practically blocked and the only relevant interlayer coupling is through the pair tunneling. Our proposed model thus becomes equivalent to Anderson's ILPT model [2] for highly underdoped systems. For the overdoped case, where $E_{g}$ is small, the ISPH within our extended model becomes appreciable with the reduction of pair tunneling process. Thus, the extended model is consistent with the implications of the transport data which predict presence of ISPH for sufficiently overdoped systems. Certain important results of the extended ILPT model are: (i) it can naturally give high values of the ratio of the superconducting gap to $T_{c}$, (ii) with increasing interlayer coupling $T_{c}$ may increase or decrease depending 
on the relative dominance of pair tunneling or ISPH, (iii) with the doping and other parameters remaining unchanged, $T_{c}$ becomes higher for materials with larger $E_{g}$, (iv) temperature varying superconducting gap data is in closer agreement with that within the extended ILPT model than the original ILPT model. These results are similar to those characterizing the high-Tc cuprate superconductors. Some of these have already been presented elsewhere [10, [1].

The presence of ISPH in our model may raise certain relevant questions regarding the issue of bilayer splitting in cuprate superconductors. The ISPH, in general, gives rise to splitting of bands which should be reflected in the electronic density of states (DOS) as well as in the spectral function, as the presence of a two peak struture. However, in recent angle resolved photoemission spectroscopy experiments (ARPES) [13], no evidence for bilayer splitting is observed in Bi2212 system. Therefore, it becomes important to investigate whether such observation is contradicted within our proposed extended model. In this communication we particularly focus on this issue. By a detailed study of the ARPES intensity curves, we show that the absence of bilayer splitting, as observed in experiments, can be understood within our proposed extended model. Our calculations are carried out including realistic band structure of Bi2212 [14] and the results are compared with the experimental data.

In the proposed extended ILPT model [10, 11] we consider the effective ISPH along with a probability factor $P$, which is suppressed by $E_{g}$. Since the microscopic origin of $E_{g}$ is still unknown and the proper functional dependence of $P$ on $E_{g}$ is difficult to predict, we make two choices for $P$. In the first one 110 we consider

$$
P=\mathrm{e}^{-E_{g} / T}
$$

which is henceforth referred to as the exponential form. In another form [1]]

$$
P=\frac{T}{E_{g}+T \mathrm{e}^{-E_{g} / T}}
$$

which will be called as T-linear form. The exponential form could signify that the charge carriers has to overcome a gap for being available for the ISPH, whereas the $T$-linear form signifies no gap, but a simple power law dependence of $P$ on $T$ and $E_{g}$. The latter 
form could find a justification within the spin-charge separation picture. Towards the end, we present arguments regarding these choices of $P$.

In this communication we also make a comparative study of the properties related to the superconducting gap with the above mentioned two different choices of the probability factor $P$. We find that, in general, the results are promising for both the choice, but the $T$-linear choice yields a better match to the experimental gap-variation data [15] than the exponential choice.

\section{Formalism}

The model hamiltonian for the coupled bilayer system is given by [10, 11]

$$
H=H_{N}+H_{S}
$$

where

$$
H_{N}=\sum_{i, k, \sigma}\left(\epsilon_{k}-\mu\right) c_{k \sigma}^{(i)+} c_{k \sigma}^{(i)}+\sum_{i \neq j, k, \sigma}\left\{t_{\perp}^{\mathrm{eff}}(k) c_{k \sigma}^{(i)+} c_{k \sigma}^{(j)}+h . c\right\}
$$

and

$$
\begin{aligned}
H_{S} & =-\sum_{i, k, k^{\prime}}\left\{V_{k, k^{\prime}} c_{k \uparrow}^{(i)+} c_{-k \downarrow}^{(i)+} c_{-k^{\prime} \downarrow}^{(i)} c_{k^{\prime} \uparrow}^{(i)}+h . c\right\} \\
& -\sum_{i \neq j, k}\left\{T_{p}^{\mathrm{eff}}(k) c_{k \uparrow}^{(i)+} c_{-k \downarrow}^{(i)+} c_{-k \downarrow}^{(j)} c_{k \uparrow}^{(j)}+h . c\right\}
\end{aligned}
$$

The operator $c_{k \uparrow}^{(i)+}\left(c_{k \uparrow}^{(i)}\right)$ is the fermion creation (annihilation) operator with momentum $k$, spin $\uparrow$ and in the layer $i(=1,2) . H_{N}$ describes the band energy of the bilayer system, where $\epsilon_{k}$ is the band dispersion in a layer, $t_{\perp}^{\text {eff }}$ is the effective interlayer single particle hopping matrix element and $\mu$ is the chemical potential. $H_{S}$ represents the interaction part of the hamiltonian and it leads to superconductivity in the system below the transition temperature $T_{c} . V_{k, k^{\prime}}$ is the in-plane pairing interaction which is responsible for the formation of Cooper pairs in a layer. $T_{p}^{\mathrm{eff}}$ represents the effective interlayer pair tunneling matrix element. For the pair tunneling term we have considered only the 
diagonal pair hopping [2], where quasi particle momentum is conserved during tunneling. Effective ISPH is taken to be $t_{\perp}^{\text {eff }}(k)=t_{\perp}^{b}(k) P$, where $t_{\perp}^{b}(k)=t_{\perp}\left(\left(\cos k_{x} a-\cos k_{y} a\right) / 2\right)^{2}$ is the $k$-dependent ISPH as predicted by the band structure calculations [3] with $t_{\perp}$ being the bare ISPH matrix element, and $P$ is the ISPH probability factor for which we consider the two forms as given in Eqs. (2) and (3). Pair tunneling, quite naturally, should be connected to the ISPH probability, since only those particles which are not taking part in ISPH, are available for the process. Tunneling of pairs, is a two particle process, hence, it occurs with a probability $(1-P)^{2}$ [10]. The effective pair tunneling matrix element is, thus, given by $T_{p}^{\mathrm{eff}}(k)=T_{p}(k)(1-P)^{2}$. The (bare) pair tunneling, following Ref. [2], is taken as $T_{p}(k)=\left[\left(t_{\perp}^{b}(k)\right)^{2} /\left|t_{1}\right|\right]$, where $t_{1}$ is the nearest neighbour hopping matrix element of the $a b$-plane band dispersion. It may be noted that for large $E_{g}$ or at very low temperatures the ISPH probability factor $P$ becomes very small within our model and all the results predicted by our model become same as that of Anderson's ILPT model. In fact, in the limit $E_{g} / T \rightarrow \infty(P \rightarrow 0)$, our model reduces exactly to the original ILPT model by Anderson.

Mean field decoupling of four fermion terms in $H_{S}$ yields

$$
H_{S}=-\sum_{i, k}\left[\Delta_{k} c_{k \uparrow}^{(i) \dagger} c_{-k \downarrow}^{(i) \dagger}+h . c\right]
$$

where $\Delta_{k}$ is the superconducting gap and is given by

$$
\Delta_{k}=\Delta_{i, k}=\sum_{k^{\prime}} V_{k, k^{\prime}}\left\langle c_{-k^{\prime} \downarrow}^{(i)} c_{k^{\prime} \uparrow}^{(i)}\right\rangle+T_{p}^{\mathrm{eff}}(k)\left\langle c_{-k \downarrow}^{(j)} c_{k \uparrow}^{(j)}\right\rangle
$$

The layers $i$ and $j$ are equivalent, since by symmetry, the in-plane pairing average is identical in both the layers.

It is well known that presence of interlayer single particle hopping between two layers produces bonding and antibonding bands. The corresponding annihilation operators are defined as $c_{k \uparrow}^{(-)}=\frac{1}{\sqrt{2}}\left(c_{k \uparrow}^{(1)}-c_{k \uparrow}^{(2)}\right)$ and $c_{k \uparrow}^{(+)}=\frac{1}{\sqrt{2}}\left(c_{k \uparrow}^{(1)}+c_{k \uparrow}^{(2)}\right)$ and $H_{N}$ becomes diagonal in this new representation. The full hamiltonian may be written in terms of these new operators as

$$
H=\sum_{i}\left[\sum_{k, \sigma} \xi_{k}^{(i)} c_{k \sigma}^{(i) \dagger} c_{k \sigma}^{(i)}-\sum_{k}\left(\Delta_{k} c_{k \uparrow}^{(i) \dagger} c_{-k \downarrow}^{(i) \dagger}+h . c\right)\right]
$$


where $i$ is now the band index and $i=-(+)$ represents the bonding (antibonding) band. Here, $\xi_{k}^{( \pm)}=\left(\epsilon_{k}-\mu\right) \pm t_{\perp}^{\text {eff }}(k)$ are the normal state band energies. From the hamiltonian of Eq.(9) the self consistent equations for the chemical potential and the superconducting gap are obtained as

$$
1-\delta=1-\frac{1}{N} \sum_{k} \xi_{k}^{(-)} \chi\left(E_{k}^{-}\right)-\frac{1}{N} \sum_{k} \xi_{k}^{(+)} \chi\left(E_{k}^{+}\right)
$$

and

$$
\Delta_{k}=\frac{\sum_{k^{\prime}} \Delta_{k^{\prime}} V_{k, k^{\prime}}\left(\chi\left(E_{k^{\prime}}^{-}\right)+\chi\left(E_{k^{\prime}}^{+}\right)\right) / 2}{1-T_{p}^{\mathrm{eff}}(k)\left(\chi\left(E_{k}^{-}\right)+\chi\left(E_{k}^{+}\right)\right) / 2}
$$

where $E_{k}^{ \pm}=\sqrt{\xi_{k}^{( \pm)^{2}}+\Delta_{k}^{2}}$, are the quasiparticle energies in the superconducting state, $\chi\left(E_{k}^{ \pm}\right)=\frac{1}{2 E_{k}^{ \pm}} \tanh \left(\frac{\beta E_{k}^{ \pm}}{2}\right), \beta=1 / T$ (in a scale of $\mathrm{k}_{B}=1$ ), $\delta=1-n$, with $\mathrm{n}$ being the number of electrons per site, and $N$ is the total number of lattice sites. The inplane attractive pairing interaction is considered here only between nearest neighbors for which $V_{k, k^{\prime}}$ is separable as $V_{k, k^{\prime}}=V \eta_{k} \eta_{k^{\prime}}$, which also makes the $k$-dependence of $\Delta_{k}$ to be separable. In our calculations, the $d_{x^{2}-y^{2}}$ symmetry of the pairing state is considered because of growing evidence of the same in high- $T_{c}$ cuprates [16, 17]. This implies $\eta_{k}=\left(\cos k_{x} a-\cos k_{y} a\right) / 2$.

Finally, the equation for the superconducting gap is obtained as

$$
\frac{1}{4 V}=\frac{1}{N} \sum_{k} \frac{\eta_{k}^{2}\left(\chi\left(E_{k}^{-}\right)+\chi\left(E_{k}^{+}\right)\right) / 2}{1-T_{p}^{\text {eff }}(k)\left(\chi\left(E_{k}^{-}\right)+\chi\left(E_{k}^{+}\right)\right) / 2}
$$

where the parameter $V$ is the nearest neighbour in-plane attractive interaction strength.

The ARPES intensity is proportional to the product of the spectral density function and the fermi distribution function. The spectral function in the superconducting state is given by

$$
A(k, \omega)=A^{-}(k, \omega)+A^{+}(k, \omega)
$$

which includes contributions from both the (bonding and antibonding) quasiparticle bands. Spectral functions for the two bands are given by

$$
A^{ \pm}(k, \omega)=\frac{1}{\pi}\left[\frac{\left(u_{k}^{ \pm}\right)^{2} \Gamma}{\left(\omega-E_{k}^{ \pm}\right)^{2}+\Gamma^{2}}+\frac{\left(v_{k}^{ \pm}\right)^{2} \Gamma}{\left(\omega+E_{k}^{ \pm}\right)^{2}+\Gamma^{2}}\right],
$$


where $\left(u_{k}^{ \pm}\right)^{2}=1-\left(v_{k}^{ \pm}\right)^{2}=\frac{1}{2}\left(1+\frac{\xi_{k}^{ \pm}}{E_{k}^{ \pm}}\right)$and $\Gamma$ is a phenomenological linewidth parameter accounting for the broadening of the quasiparticle states due to finite lifetime.

Considering the experimental energy resolution factor the intensity of the ARPES is given by [18]

$$
I(k, \omega)=I_{0} \int_{-\infty}^{\infty} R\left(\omega-\omega^{\prime}\right) f\left(\omega^{\prime}\right) A\left(k^{\prime}, \omega^{\prime}\right) d \omega^{\prime}
$$

where $R(\omega)$ is the Gaussian energy resolution function and $f(\omega)=1 /\left(e^{\beta \omega}+1\right)$ is the fermi distribution function.

\section{Results and Discussion}

A relevant point of contention, that may be raised regarding the validity of our model, as proposed in Ref. [10, 11, is that in experiments no evidence of bilayer splitting is observed in Bi2212. This suggests that the interlayer single particle hopping would be absent, while a finite ISPH is present in our model. So, at first we present the results about the ARPES intensity within our model. By numerical solution of the self-consistent equations (10) and (12) we evaluate the superconducting gap as a function of temperature and hence determine the spectral density function as well as the ARPES intensity in the superconducting phase. For the in-plane band dispersion we consider a six parameter tight binding band structure, $\left[t_{0}, t_{1}, t_{2}, t_{3}, t_{4}, t_{5}\right]=[0.131,-0.149,0.041,-0.013,-0.014,0.013]$ $e V$, where $t_{0}$ is the bare Wannier orbital energy, $t_{1}$ nearest neighbor, $t_{2}$ next nearest neighbor etc. hopping matrix elements, as described [14] and used in previous publications [19]. We take $t_{\perp}=40 \mathrm{meV}$ and the in-plane attractive interaction $V=70 \mathrm{meV}$, which are reasonable. To begin with, we consider the ARPES intensity and single particle DOS for the exponential choice of $P$.

The ARPES intensity $I(k, \omega)$ has been calculated using Eqs.(13)-(14) at the Brillouin zone point $k=(\pi, 0)$ for optimal doping and for different values of the energy resolution characterised by the full width at half maximum (FWHM) values of the Gaussian energy resolution function. The value of $E_{g}$ at optimal doping is taken from a best fit analysis 
of the theoretical curve for the temperature variation of the superconducting gap to the experimental data, which is presented later. The plots of $I(k, \omega)$ versus $\omega$ at different temperatures are shown in Fig.1, where we take a very small value for the linewidth parameter $\Gamma \sim 1 \mathrm{meV}$. A perfect energy resolution is assumed in Fig.1a. The figure shows that even within the limit of perfect resolution $(\mathrm{FWHM}=0)$ no bilayer splitting could be observed at $\mathrm{T}=13 \mathrm{~K}$, while at $\mathrm{T}=40 \mathrm{~K}$ a splitting is observable in $I(k, \omega)$, but the difference in energy between the two peaks is only $\sim 8 \mathrm{meV}$. Fig. 1b shows the evolution of $I(k, \omega)$ at $\mathrm{T}=40 \mathrm{~K}$ from a two peak structure to a single peak structure as the FWMH of the energy resolution function increases from $2 \mathrm{meV}$ to $10 \mathrm{meV}$. The experimental energy resolution for the ARPES, as quoted by different workers [18, 20], is $\sim 19 \mathrm{meV}$, which is larger than that we have considered. Furthermore, at $T=40 \mathrm{~K}$ the linewidth parameter in cuprates would be much larger than $1 \mathrm{meV}$ [21], which will further broaden the spectral function and hence the $I(k, \omega)$. So, it is clear that no bilayer splitting could be observed in the ARPES experiments within our model for the parameter space, relevant for high- $T_{c}$ cuprates, and for the exponential choice of $P$.

Presence of the ISPH in a bilayer system would also produce a splitting in the normal state electronic DOS. So a study of the normal state DOS could provide a way to examine the significance of the bilayer splitting. In Fig.2 we plot the electronic DOS at different temperatures and for different values of the linewidth parameters $\Gamma$. It may be noted that the effective ISPH in our model is temperature dependent and it makes the DOS to be dependent on temperature. Fig.2a shows the plot of the DOS at $T=10 \mathrm{~K}$. It is found that even with $\Gamma=0$, no evidence of bilayer splitting in the DOS could be observed within our model for the exponential choice of $P$ at very low temperatures. For $T=$ $50 \mathrm{~K}$, bilayer splitting is quite evident for $\Gamma=0$. However, as the value of $\Gamma$ is increased to $14 \mathrm{meV}$ only one broad peak is observed in the DOS. For high- $T_{c}$ cuprates the value of $\Gamma$ is quite large at high temperatures [21]. Thus, our study shows that bilayer splitting may not be observable experimentally even if the normal state DOS is probed directly.

In Fig. 3 we present the plot of the intensity of the ARPES $(k=\pi, 0)$ for the $T$-linear form of $P$ and for a value of the linewidth parameter $\Gamma=1 \mathrm{meV}$. For this form of 
$P$ the suppression of the effective ISPH by $E_{g}$ at low temperatures is much less rapid than the exponential form of $P$. Consequently, at $T=13 K$ the $I(k, \omega)$ shows a two peak structure when the energy resolution is perfect $(\mathrm{FWHM}=0)$. However, even for FWHM as small as $3 \mathrm{meV}$ the $I(k, \omega)$ takes a single peak form at $T=13 \mathrm{~K}$. Fig. $3 \mathrm{~b}$ shows how the two peak form of the $I(k, \omega)$ at $T=40 \mathrm{~K}$ evolves into a single peak with decreasing energy resolution or increasing value of FWHM of the Gaussian energy resolution function. A single peak form for $I(k, \omega)$ is obtained at $T=40 K$ for a value of $\Gamma$ as small as $1 \mathrm{meV}$ and a value of FWHM of the energy resolution function as $12 \mathrm{meV}$, which is less than the FWHM values of the resolution function in experiments [18, 20]. It should be noted that consideration of higher values of $\Gamma$ would produce increased broadening in the shape of the $I(k, \omega)$, hence a single peak form would be obtained even with a better energy-resolution (a lower value of FWHM) in actual experiments.

In previous works [10, 11] we studied the superconducting phase diagram and some of the superconducting properties within our model for different $E_{g}$ values. The value of $E_{g}$ changes with doping in cuprates. However, for optimal doping $E_{g}$ has a fixed value for any particular cuprate material. In this communication we study different superconducting properties only at optimal doping and make a comparison of the results, obtained with two choices of $P$, with experimental data. In Fig.4 we present the best-fit curves for the temperature variation of the superconducting gap for two choices of $P$ alongwith the experimental data points. The curve for the Anderson's pair tunneling model $\left(E_{g}=\infty\right)$ is also shown. It is clearly seen that predictions within our model are much closer to the experimental data than that with the original ILPT model. For the exponential choice of $P$, it is found that $E_{g} \sim 6-7 \mathrm{meV}$ gives a very good fit to the experimental data of Bi2212 at optimal doping. This value of $E_{g}$ is very close to the transition temperature of Bi2212 system at optimal doping. It is interesting to note that the value of $E_{g} \sim T_{c}$ at optimal doping has been suggested in cuprates from experimental observations also [9].

However, with the exponential form of $P$ we find that it is difficult to obtain good fit to the experimental data points for the gap variation at low temperatures. A precise fit 
to the experimental data is possible (Fig.4) for the second choice of $P$, where it increases almost linearly with temperature (Eq.(3)). However, the value of $E_{g}$, required for such a fit is higher than that for the exponential choice of $P$. It should be noted that due to the absence of any microscopic derivation for the correct form of $P$, we make possible choices which are purely phenomenological. In the exponential choice, $E_{g}$ appears as a gap to hinder the charge transport along the $c$-axis. But, in the $T$-linear choice there is no direct gap and the conditions that $P$ in Eq.(3) has to satisfy, are: (i) $P$ increases linearly with $T$ while decreases with $E_{g}$ for $T \ll E_{g}$ and (ii) $P=1$ for $E_{g}=0$. Clearly, these conditions could be satisfied even if a prefactor is added to $E_{g}$. Thus the value of $E_{g}$, as in Eq.(3), may differ from the actual value by a constant factor.

In high- $T_{c}$ systems the ratio of the superconducting gap to $T_{c}$ is very high compared to the conventional superconductors. The value of the gap ratio $\left(2 \Delta_{0} / T_{c}\right)$ in cuprates is reported to be within the range 6-9 [21]. In Fig.5 we plot the gap ratio as a function of interlayer single particle hopping matrix element $\left(t_{\perp}\right)$ for two different values of $E_{g}$ and for both the forms of $P$, for a doping level which is optimal for $t_{\perp}=40 \mathrm{meV}$. The ratio increases with increasing value of $t_{\perp}$. It is seen that both choices of $P$ yield quite high values of the gap ratio, but for the $T$-linear choice of $P$ the ratio is larger. For $t_{\perp}=40 \mathrm{meV}$, which is realistic for layered cuprates, the value of the gap ratio lies within the range 5.0-7.5 (depending on $E_{g}$ and the choice of $P$ ). Thus, our model could yield high values for the gap ratio in agreement with the experimental findings in cuprates.

It is observed that the gap ratio is higher for a smaller value of $E_{g}$ (Fig.5). Within our model a lower value of $E_{g}$ yields a lower value for $T_{c}^{m}$ (transition temperature at optimal doping) [10] and this is consistent with the experimental findings [9]. The results of Fig. 5, thus, predict a higher value of the gap ratio for a cuprate system with lower value of $T_{c}^{m}$.

As found in the results presented above that the qualitative characteristics of them do not depend much on the specific form of the probability factor $P$ used. While making a choice, one only needs to ensure that the probability factor $P$, and as a result the effective ISPH, follows the observed doping dependence of $c$-axis transport, that is 
the effective $c$-axis hopping remains heavily suppressed in the underdoped side and at low temperatures. In experiments, $E_{g}$ is found to grow in magnitude [7, 8 towards underdoing. Thus $E_{g}$, on phenomenological grounds, could be taken to suppress $c$-axis hopping, and hence our choices of $P$ could follow.

It should be mentioned that, an exponential suppression of effective c-axis hopping towards underdoping was suggested [0] from the experimental results in Y-123 system where a metallic like behavior of c-axis resistivity at high temperatures and a semiconducting behavior at low temperatures have been observed. But, our calculations show that the $T$-linear choice of $P$ gives a better match to the experimental gap-variation data. One could probably search for a realization of this $T$-linear choice within within the RVB picture [12]. According to the RVB model, holons and spinons are the quasiparticles in a layer of strongly correlated system and a holon has to combine with a spinon to form a hole which can then hop from one layer to another. Consequently, $c$-axis hopping is proportional to the spinon density, which increases linearly with $T$ within the RVB theory and hence, the effective $c$-axis single particle hopping is expected to be proportional to $T$.

\section{Conclusions}

Investigation of the spectral density function and corresponding intensity curves for the ARPES within our proposed extendeded pair tunneling model shows that the model has no contradiction with the absence of bilayer splitting observed in Bi2212 by ARPES experiment [13]. Studies with two different forms for the probability factor $P$, associated with the effective interlayer single particle hopping, show that both the forms could yield high values for the ratio of the superconducting gap to $T_{c}$, as observed in cuprates. Temperature varying superconducting gap from our calculations with both the forms

agrees well with the experimental data and the agreement is much better compared to that within the original ILPT model. A precise fit to the experimental gap variation 
data is achieved with the $T$-linear choice of $P$. Another prediction of our model is that a system which has a lower value of $E_{g}$ should have a lower value of $T_{c}^{m}$ and a larger value of the gap ratio.

It may be mentioned that Anderson's ILPT model is applicable in the underdoped region where non-fermi liquid behavior dominates and the ISPH is negligible, whereas our extended model has the flexibility of being applicable in a wide region from the underdoped to a highly overdoped regime where the ISPH is appreciable.

In the model, presented here, we have considered the effect of $E_{g}$ only on the interlayer hopping. However, it seems natural to think that, $E_{g}$ should also affect the in-plane charge dynamics. In this connection, it has been suggested that $E_{g}$ reduces the in-plane electronic density of states [9]. It would be interesting to study the extended model with the pseudogap $E_{g}$ affecting both the in-plane and out of plane charge dynamics. This would constitute future communications. 


\section{References}

[1] P. W. Anderson, in Superconductivity, Proceedings of the ICTP spring college in 1992, eds. P. Butcher and Y. Lu (World Scientific, Singapore); Science 256 (1992) 1526.

[2] S. Chakravarty, A. Sudbo, P. W. Anderson and S. Strong, Science 261, (1993) 337.

[3] O. K. Andersen, A. I. Liechtenstein, O. Jepsen and F. Paulsen, J. Phys. Chem. Solids 56, (1995) 1573.

[4] K. Tamasaku, Y. Nakamura and S. Uchida, Phys. Rev. Lett. 69, (1992) 1455; B. Batlogg, in High Temperature Superconductivity, eds. K. S. Bedell et al. (AddisonWesley, 1990).

[5] S. L. Cooper and K. E. Gray, in Physical Properties of High Temperature Superconductors, Vol.IV, ed. D. M. Ginsberg (World Scientific, 1994).

[6] S. Uchida, K. Tamasaku and S. Tajima, Phys. Rev. B 53, (1996) 14558 and refrences therein.

[7] A. G. Loeser et al., Science 273, (1996) 325.

[8] H. Ding et al., Nature 382, (1996) 512.

[9] J. W. Loram et al., J. Supercond. 7, (1994) 243; G. V. M. Williams et al., Phys. Rev. Lett. 78, (1997) 721.

[10] A. N. Das and S. Sil, Physica C 299, (1998) 83.

[11] B. Chattopadhyay and A. N. Das, Phys. Lett. A (in press).

[12] P. W. Anderson and Z. Zou, Phys. Rev. Lett. 60, (1988) 132.

[13] H. Ding et al., Phys. Rev. Lett. 76, (1996) 1533. 
[14] M. R. Norman et al., Phys. Rev. B 52, (1995) 615.

[15] M. Itoh, S. Karimoto, K. Namekawa and M. Suzuki, Phys. Rev. B 55, (1997) 12001.

[16] W. N. Hardy et al., Phys. Rev. Lett. 70, (1993) 3999; D. A. Brawner and H. R. Ott, Phys. Rev. B 50, (1994) 6530; J. R. Kirtley et al., Nature (London) 373, (1995) 225; C. C. Tseui et al., Nature 387, (1997) 481.

[17] Z. X. Shen et al., Phys. Rev. Lett. 70, (1993) 1553; H. Ding et al., Phys. Rev. B 54, (1996) 9678.

[18] H. Ding et al., Phys. Rev. Lett. 74, (1995) 2784.

[19] B. Chattopadhyay, D. Gaitonde and A. Taraphder, Europhys. Lett. 34, (1996) 705; B. Chattopadhyay, Phys. Lett. A 226, (1997) 231; B. Chattopadhyay, J. Lahiri and A. N. Das, Mod. Phys. Lett. B 11, (1997) 1285.

[20] Z. X. Shen and J. R. Schrieffer, Phys. Rev. Lett. 78, (1997) 1771.

[21] T. Hasegawa, H. Ikuta and K. Kitazawa, in Physical Properties Of High Temperature Superconductors, Vol.III, ed. D. M. Ginsberg (World Scientific, 1992). 


\section{Figure captions:}

Fig.1. Plot of the ARPES intensity $I(k, \omega)$ in arbitrary units versus $\omega(\mathrm{eV})$ for the exponential choice of $P$. Different values of FWHM of the Gaussian energy resolution function and temperatures are as given in the figure. The linewidth parameter is taken to be $\Gamma=1 \mathrm{meV}$.

Fig.2. Plot of the electronic density of states $N(\xi)$ versus $\xi$ for the exponential choice of $P$. Temperatures and different values of linewidth parameter $\Gamma$ are mentioned in the figure.

Fig.3. The ARPES intensity $I(k, \omega)$ versus $\omega$ for the $T$-linear choice of $P$ with the linewidth parameter $\Gamma=1 \mathrm{meV}$. Values of FWHM and temperatures are listed in the figure.

Fig.4. Finite temperature gap scaled to its zero temperature value $\left(\Delta_{k}^{\max }(T) / \Delta_{k}^{\max }(0)\right)$, as a function of reduced temperature $\left(T / T_{c}\right)$. Solid line is that in the Anderson limit $\left(E_{g}=\infty\right)$, solid square symbols are experimental data from Ref. 115 and dashed lines are from our calculations for two different forms of the probability factor $P$.

Fig.5. Maximum value of the gap-ratio $\left(2 \Delta_{k}^{\max }(0) / T_{c}\right)$ versus bare interlayer coupling $\left(t_{\perp}\right)$, for different $E_{g}$ values and different choices of $P$, as mentioned in the figure. 


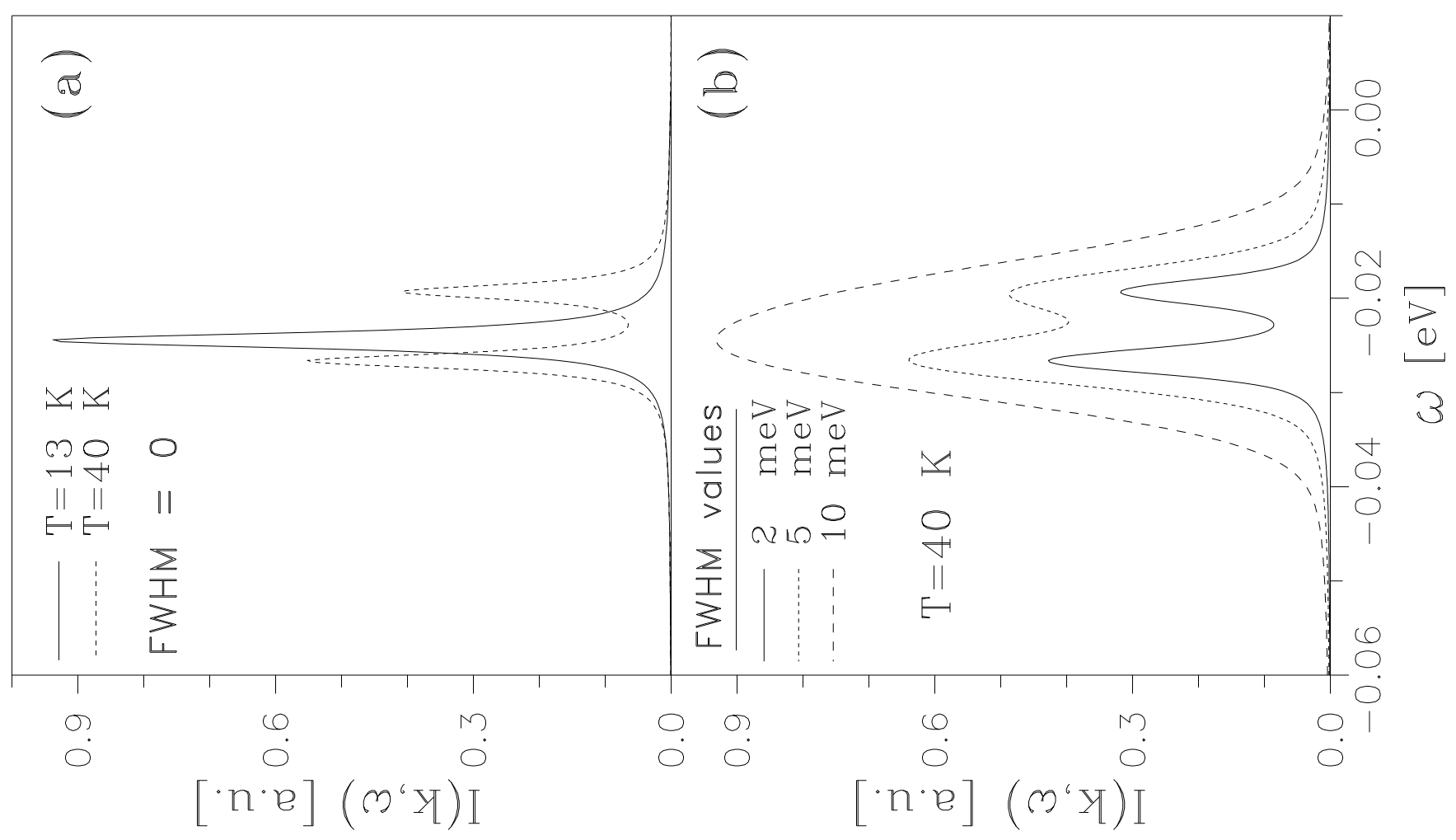




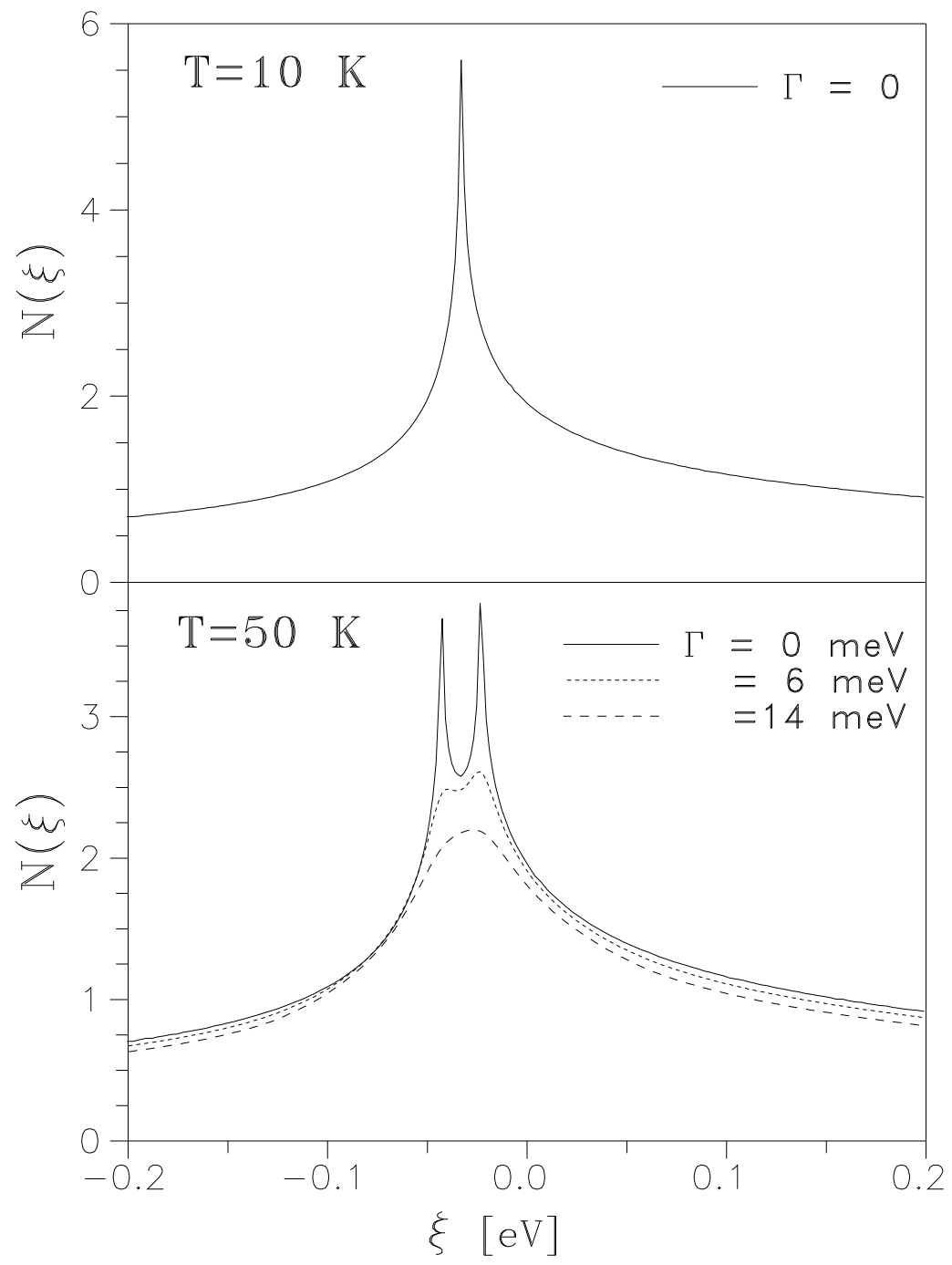




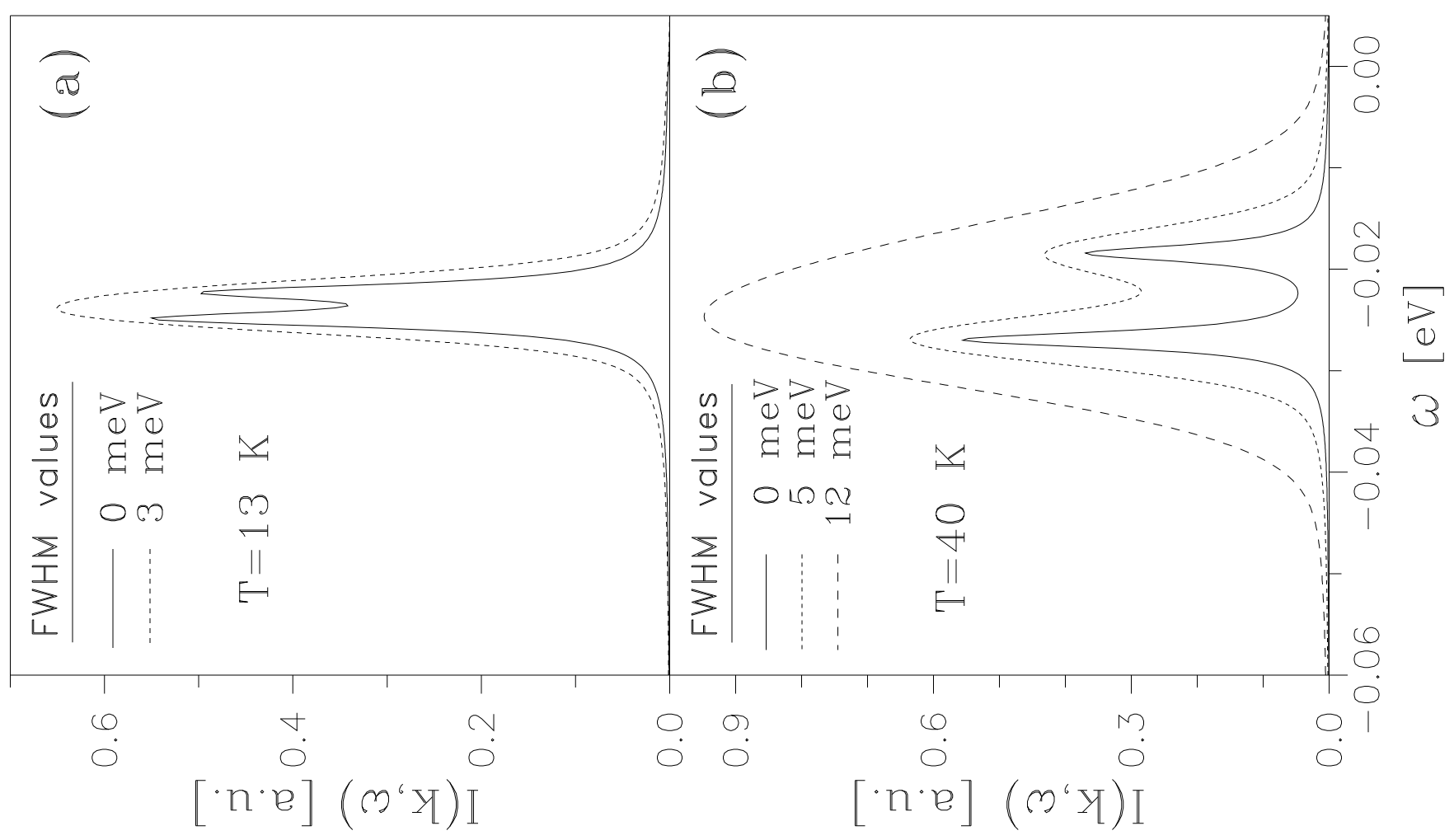




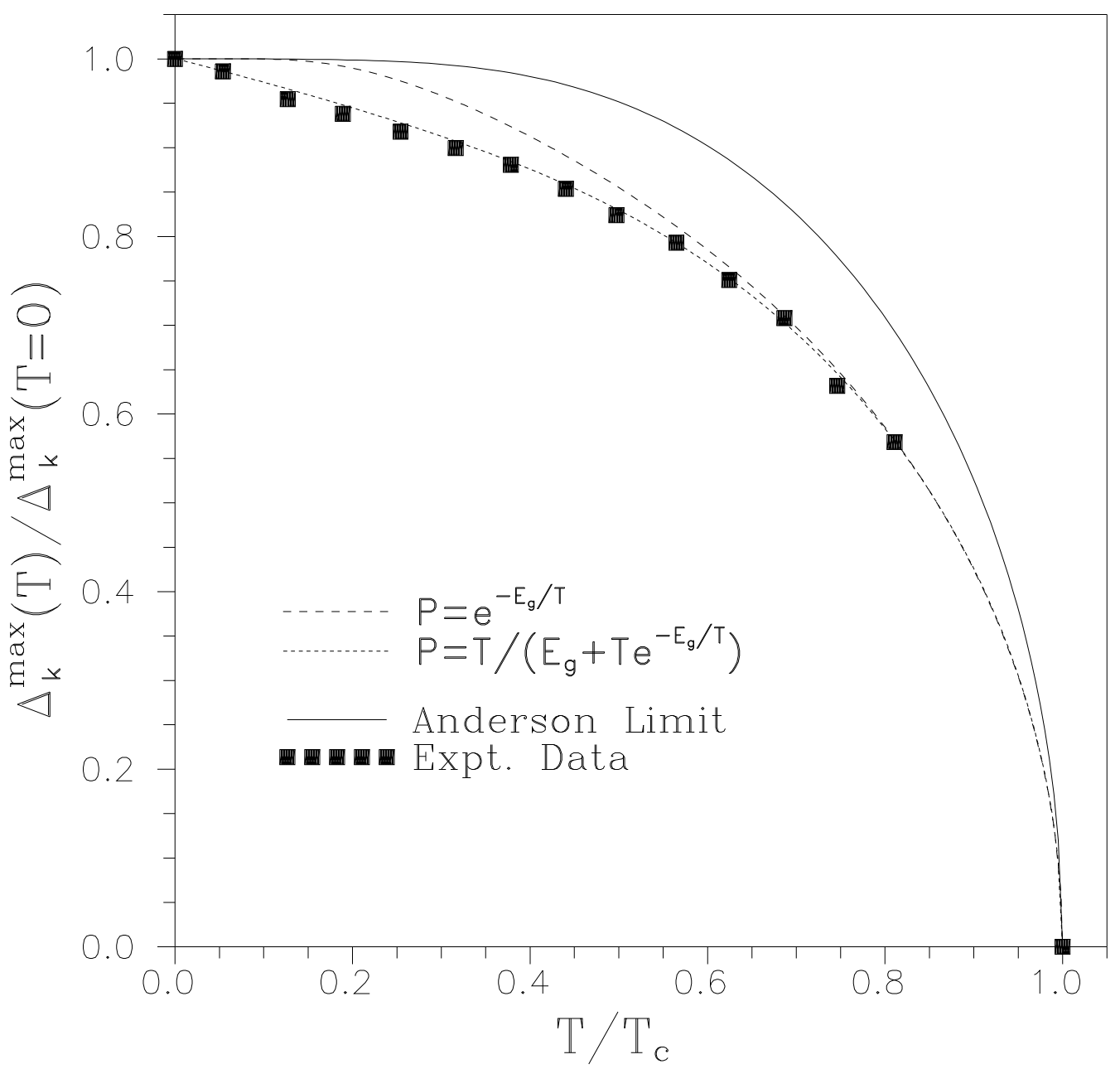




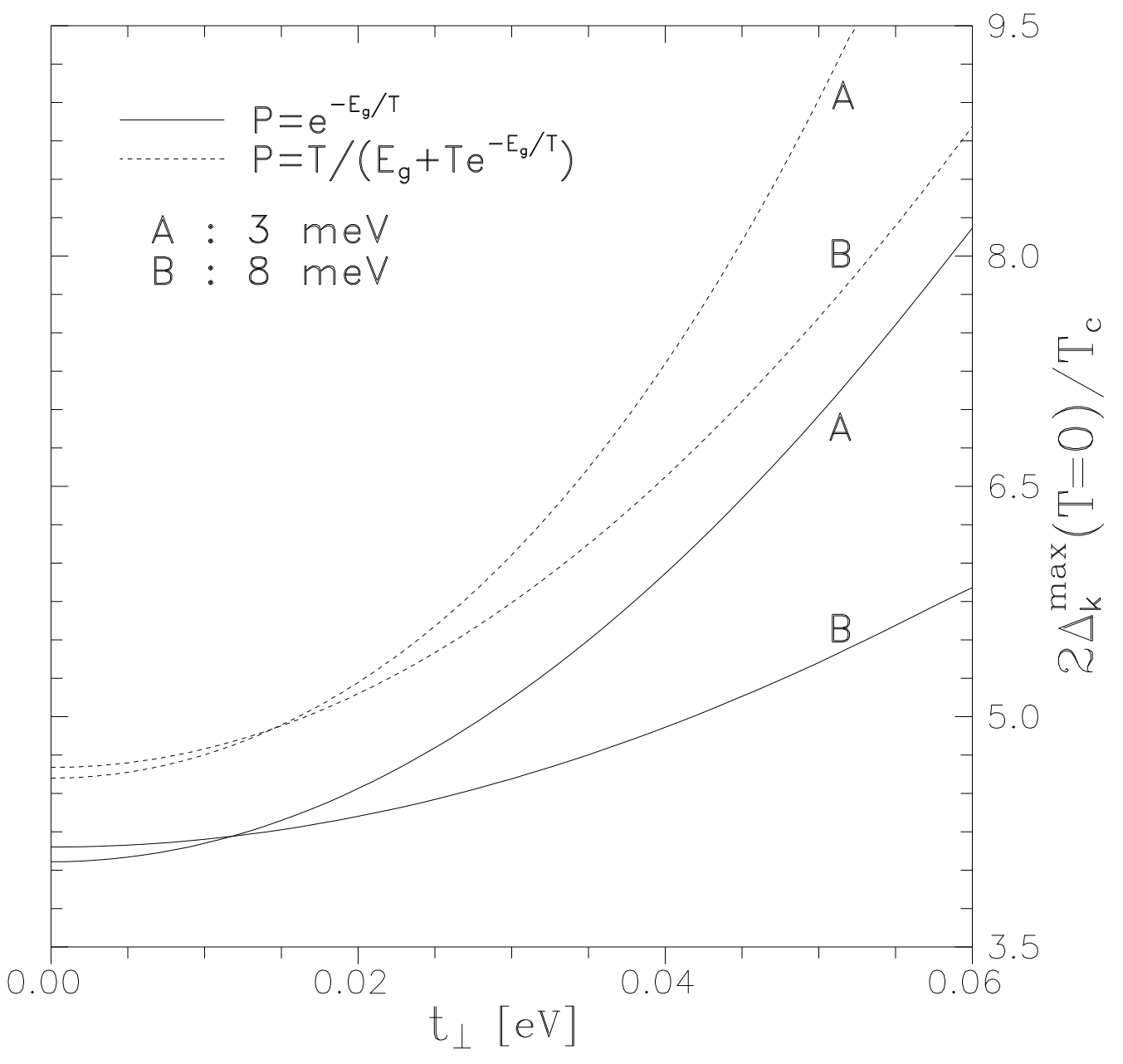

